\title{
ECOEFICIÊNCIA: UMA ANÁLISE DAS METODOLOGIAS DE MENSURAÇÃO E SEUS RESPECTIVOS INDICADORES
}

\author{
Luciano Munck \\ Doutor em Administração Universidade de São Paulo - USP \\ Professor Universidade Estadual de Londrina - UEL \\ munck@uel.br \\ Flávio Augusto Cella-de-Oliveira \\ Mestrando em Administração da Universidade Estadual de Londrina - UEL \\ flaviocella@yahoo.com.br \\ Ana Claudia Bansi \\ Mestranda em Administração da Universidade Estadual de Londrina - UEL \\ ana_bansi@hotmail.com
}

\begin{abstract}
RESUMO
Os indicadores utilizados para mensurar a ecoeficiência ainda não estão consolidados e, em sua maioria, apresentam aplicação inviável para as organizações. Nesse sentido, emergiu a necessidade de uma análise crítica das metodologias de mensuração e seus respectivos indicadores. Para esta pesquisa, foram selecionados dois grupos de indicadores que apresentam considerável significância para a comunidade prático/acadêmica: os indicadores do World Business Council for Sustainable Development - WBCSD e do Global Reporting Initiative - GRI. Nesse contexto, este artigo teve como objetivo analisar as possíveis fragilidades destes indicadores, utilizando-se, como parâmetro, os Princípios de Bellagio, que sintetizam a percepção geral sobre os principais aspectos relacionados aos indicadores da sustentabilidade. Como resultados principais, este estudo mostrou que os indicadores pesquisados, diante dos parâmetros de análises utilizados, não possuem consistência suficiente para afirmar que mensuram a ecoeficiência de forma eficaz. Todavia, servem como subsídios para criação de indicadores adaptados ao contexto de cada organização.
\end{abstract}

Palavras-chave: Ecoeficiência; Indicadores de sustentabilidade; Relatórios de sustentabilidade.

\section{ECO-EFFICIENCY: A REVIEW OF METHODS OF MEASUREMENT AND THEIR INDICATORS}

\begin{abstract}
A preliminary analysis showed that the indicators used to measure the eco-efficiency are not yet consolidated, and mostly present application impractical for organizations. In this sense, it's necessary a review of measurement methodologies and their respective indicators. If measures cause inconsistencies disjointed decisions it's another justification for this study. For the survey were selected two groups of indicators that have considerable practical significance for the community/academic: Indicators of the World Business Council for Sustainable Development WBCSD and the Global Reporting Initiative - GRI. This article aims to analyze the possible weaknesses of these indicators using as parameter the Bellagio Principles, which summarize the general perception of major issues related to indicators of sustainability. As main results, the study showed that the indicators studied, before the analysis parameters used, have not consistently enough to effectively measure eco-efficiency. However, they serve as start point to create new consistent indicators and adapted to the context of each organization.
\end{abstract}

Key words: Eco-efficiency; GRI; Indicators; Measurement; Wbcsd.

Revista de Gestão Social e Ambiental - RGSA, São Paulo, v. 5, n. 3, p. 183-199, set./dez. 2011. 


\section{INTRODUÇÃO}

As discussões inerentes às causas ambientais já alcançam meio século de vida, contudo, foi na última década que houve a acentuação desse debate. No contexto organizacional, este tema está presente, devido às imposições legais. Contudo, o grande desafio é ultrapassar as barreiras legais, fazendo com que as organizações incorporem os princípios do desenvolvimento sustentável em seus valores.

Em meio a esta discussão da sustentabilidade, emerge a ecoeficiência, que, para o World Business Council for Sustainable Development - Wbcsd (2000a), reúne os ingredientes essenciais progresso econômico e ambiental - para o aumento da prosperidade econômica, por meio da utilização mais eficiente dos recursos e de menos emissões nocivas para o ambiente, procurando melhorias ambientais que potencializem, paralelamente, benefícios econômicos. Basicamente, essa iniciativa concentra-se em oportunidades de negócio e permite às empresas tornarem-se mais responsáveis, do ponto de vista ambiental, e mais lucrativas, além de incentivar a inovação e, por conseguinte, o crescimento e a competitividade (Wbcsd, 2000b). Em meio à hegemonia do pilar econômico, se a eficiência econômica for unida à eficiência ambiental, leva a ecoeficiência (Bleischwitz, 2003). Nesse contexto, diz-se que a ecoeficiência é parte integrante do movimento da sustentabilidade organizacional (Savitz \& Weber, 2007).

De acordo com Krajnc e Glavic (2005), muitas empresas que até então consideravam apenas os indicadores financeiros para acompanhar os resultados de suas atividades, passaram a desenvolver práticas direcionadoras à sustentabilidade e à ecoeficiência, mas como a perspectiva econômica, a ecoeficiência precisa ser mensurada, por isso, se utilizam indicadores, que são informações úteis para a tomada de decisões, que refletem informações e parâmetros sobre um determinado fenômeno, auxiliando na identificação de lacunas entre os objetivos, estratégias e programas da instituição.

Huppes e Ishikawa (2005) afirmam que o "assunto ecoeficiência" mostra-se muito diverso, ou rico de atribuições. Apesar da dificuldade que esta diversidade confere ao objeto de estudo, VanBellen (2004) supõe que tal diversidade de conceituação deve servir não como obstáculo, mas sim como fator de motivação e de criação de novas visões acerca de ferramentas que procurem descrever a sustentabilidade, e, no contexto deste artigo, a ecoeficiência. Desta forma, o desenvolvimento de indicadores é importante para que seja possível mensurar o desenvolvimento da ecoeficiência nas organizações, e a partir de então poder utilizar-se de diferentes abordagens para melhor desenvolvê-la.

A importância dos indicadores para as organizações se define como uma maneira de organizar uma vasta quantidade de informações sobre uma ampla variedade de riscos e oportunidades (Savitz \& Weber, 2007). Kranjc e Glavic (2003) consideram os indicadores importantes porque eles resumem uma grande quantidade de informações de diferentes fontes em um formato fácil para entender, comparar e manipular. Pensando nas organizações, os indicadores podem contribuir por ajudar o tomador de decisão a visualizar quais ações precisam ser enfatizadas no futuro.

Contudo, em razão da complexidade de mensurar aspectos relacionados com a sustentabilidade, prevalecem a confusão e a arbitrariedade conceitual nos indicadores de desempenho ambiental. Para Callado e Fensterseifer (2009), a abundância de iniciativas e métricas de sistemas de indicadores de sustentabilidade, e, ainda, mediante o grande avanço no número de pesquisas, os indicadores que mensuraram a sustentabilidade, assim como a ecoeficiência, ainda não estão consolidados cientificamente. Kranjc e Glavic (2003) citam que, apesar do surgimento de muitos indicadores, a maioria deles não é aplicável.

Azapagic e Perdan (2000), tratando das fragilidades dos indicadores de sustentabilidade, afirmam que estão em desenvolvimento e que há poucos exemplos quantitativos de suas aplicações. Além disso, os autores destacam que algumas abordagens são muito complicadas, uma vez que incluem grande número de indicadores que se tornam difíceis de quantificar ou compreender,

Revista de Gestão Social e Ambiental - RGSA, São Paulo, v. 5, n. 3, p. 183-199, set./dez. 2011. 
aumentando a dificuldade de sua aplicação prática, e, consequentemente, não orientando eficazmente o processo de tomada de decisão.

Desta forma, evidencia-se a necessidade de uma análise crítica e acurada das metodologias de indicadores existentes. Assim, tendo em mente a importância dos indicadores bem como suas fragilidades, foram selecionados dois indicadores para análise, a partir de sua relevância, identificada pela constante presença em artigos que tratam do assunto: os indicadores do World Business Council for Sustainable Development - WBCSD e do Global Reporting Initiative - GRI. Portanto, este artigo tem como objetivo analisar a robustez dos indicadores de ecoeficiência, assim como possíveis fragilidades existentes nestes modelos escolhidos, com o intuito de servir como diretrizes para a construção de novos modelos que possam aproximar-se de uma mensuração mais eficiente.

Para uma melhor compreensão do tema em investigação, este artigo foi desenvolvido com a apresentação das seguintes seções: introdução; metodologia; revisão bibliográfica, contemplando discussões em torno do desenvolvimento sustentável e sustentabilidade organizacional, ecoeficiência, indicadores de forma geral e de ecoeficiência; análise dos resultados; e considerações finais.

\section{FUNDAMENTAÇÃO TEÓRICA}

Para o desenvolvimento dessa proposta, estruturou-se a presente fundamentação teórica, que inicia o debate sobre desenvolvimento sustentável e a sustentabilidade organizacional, passando pela ecoeficiência e seus indicadores.

\subsection{Desenvolvimento sustentável e sustentabilidade organizacional}

O termo desenvolvimento sustentável tornou-se conhecido em 1987, no Relatório Brundtland, publicado no livro Our Common Future, elaborado pela Wced - World Commission on Environment and Development, que o define como "[...] o desenvolvimento que procura satisfazer as necessidades da geração atual, sem comprometer a capacidade das gerações futuras de satisfazerem as suas próprias necessidades" (Wced, 1987). A expressão "desenvolvimento sustentável" foi cunhada com o objetivo de incluir os processos decisórios e as políticas baseadas na interdependência e complementaridade de crescimento econômico e preservação do meio ambiente (Osorio, Lobato \& Castillo 2005).

Embora esta definição passe a ser aceita por muitos autores e documentos, esta ideia de desenvolvimento sustentável é alvo de constantes críticas (Hoff, 2008; Laville, 2009), em razão da dificuldade de delimitação ou caracterização das necessidades que ele pressupõe que devam ser atendidas, além das formas de mensuração empregadas. Algumas destas críticas são apontadas por Hoff (2008) a respeito do conceito de equidade entre a geração futura e atual.

Tendo em vista as críticas ao conceito de desenvolvimento sustentável proposto no Relatório Brudtland, é necessário entender a sustentabilidade organizacional, por meio da qual a ecoeficiência ganha notoriedade. Assim, a sustentabilidade, para Souza (2010), é traduzida como a ideia central perseguida pelo desenvolvimento sustentável, já que a origem, os espaços, os períodos e os contextos de um determinado sistema se integram em um processo contínuo de desenvolvimento.

Entretanto, embora os conceitos de desenvolvimento sustentável e de sustentabilidade sejam frequentemente abordados de maneira análoga, conforme tratam Munck e Souza (2009a), cada um indica uma situação diferente. Sustentabilidade é um estado, em que uma organização ou sociedade encontra-se em relação aos aspectos econômicos, ambientais e sociais, logo, ao se dizer que uma organização ou uma sociedade é sustentável deseja-se afirmar que esta é detentora de um determinado nível de sustentabilidade. Munck e Souza (2009b) complementam que o desenvolvimento sustentável e a sustentabilidade buscam os mesmos objetivos, mas, o desenvolvimento sustentável deve ser considerado como um caminho para a sustentabilidade.

No atual debate da sustentabilidade, este artigo destaca a sustentabilidade organizacional,

Revista de Gestão Social e Ambiental - RGSA, São Paulo, v. 5, n. 3, p. 183-199, set./dez. 2011. 
que se preocupa em responder tais problemas em âmbito organizacional. Assim, por uma perspectiva de compreensão extraída dos estudos organizacionais, Silva e Quelhas (2006) conceituam o desenvolvimento sustentável como um tipo diferenciado de desenvolvimento que busca por um equilíbrio entre o que é socialmente desejável, economicamente viável e ecologicamente sustentável. Percebe-se, assim, que a legitimação dos lucros empresariais por uma aceitação social e comunitária, passa a ser imprescindível.

A sustentabilidade organizacional, para Dyllick e Hockerts (2002), pode ser definida como a capacidade de as empresas alavancarem seus capitais, econômico, social e ambiental, para contribuir com o desenvolvimento sustentável em seu domínio político. De tal modo, a sustentabilidade organizacional emerge da mudança no ambiente das organizações: as empresas que eram vistas como instituições apenas econômicas têm presenciado o surgimento de novos papéis que devem ser desempenhados, como resultado das alterações no ambiente em que operam (Donaire, 2009), e, neste contexto, a sustentabilidade organizacional tem ganhado espaço nos debates.

O conceito do Triple Bottom Line - TBL indica a interação entre os resultados financeiros, ambientais e sociais na mensuração do desempenho corporativo, e assim, ressalta que a sustentação dos resultados das empresas no longo prazo depende da conservação e do desenvolvimento das diversas formas de capital (Lemme, 2010). Tal modelo divide sustentabilidade em três pilares fundamentais, sendo estes, o pilar econômico, ambiental e social. A principal evidência deste modelo é que a organização não deve basear suas decisões em apenas um dos pilares, pois, conforme Laville (2009). A Figura 1 possibilita uma melhor visualização do processo da sustentabilidade em âmbito organizacional.

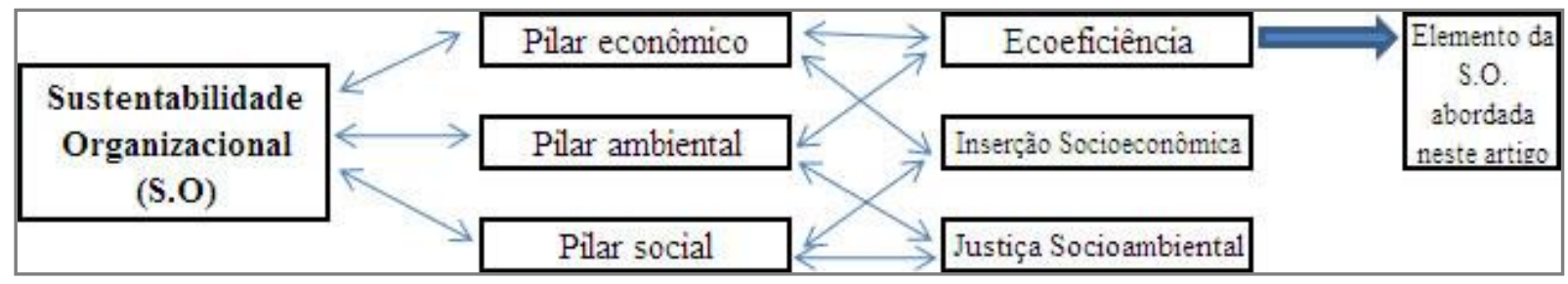

Figura 1: Sustentabilidade organizacional

Fonte: Elaborado a partir de Elkington (1999), Savitz e Weber (2007) e Munck, Munck e Souza (2011).

Como mostra a Figura 1, ao falar de sustentabilidade organizacional deparamo-nos com o modelo baseado em Savitz e Weber (2007) e Elkington (1999). Assim como Souza (2010) e Munck, Munck e Souza (2011) visa a integração dos três pilares capazes de, juntos, proporcionar um estado de sustentabilidade organizacional, a saber: sustentabilidade econômica, ambiental e social. O embasamento desta construção da sustentabilidade organizacional por meio das três sustentabilidades menores pode ser encontrado em diversos autores, como Dillick e Hockerts (2002) e Kranjc e Glavic (2003), assim como Callado (2010).

Com base na Figura 1 e analisando as três sustentabilidades, para ser considerada sustentável, uma organização deve, antes, desenvolver suas sustentabilidades econômica, ambiental e social. Segundo Elkington (1999) e Savitz e Weber (2007) a conexão entre as sustentabilidades organizacionais (econômica, ambiental e social), duas a duas, resultam em fenômenos de relacionamento, que permitem as organizações trabalhar os três pilares, a saber:

- Inserção socioeconômica (conexão da sustentabilidade econômica e social): engajar a organização em favor do coletivo, de modo que todos tenham acesso à informação, alimentação, saúde, educação, habitação, trabalho, renda e dignidade;

- Justiça socioambiental (conexão das sustentabilidade social e ambiental): é a equalização da distribuição dos benefícios e dos constrangimentos impostos pela legislação ambiental, ou mesmo pelos problemas ambientais, entre diferentes grupos

Revista de Gestão Social e Ambiental - RGSA, São Paulo, v. 5, n. 3, p. 183-199, set./dez. 2011. 
sociais;

- Ecoeficiência (conexão da sustentabilidade econômica e ambiental): é a busca pelo aumento da prosperidade econômica, por meio da utilização mais eficiente dos recursos e de menos emissões nocivas para o ambiente.

Embora os três fenômenos citados, assim como as três sustentabilidades organizacionais, tratem de perspectivas diferentes do contexto organizacional, enfatiza-se a necessidade de que sejam desenvolvidas, simultaneamente, para o alcance da sustentabilidade organizacional. Contudo, independentemente da motivação, o desenvolvimento da sustentabilidade organizacional parece ser uma tendência das organizações. Bleischwitz (2003) propõe um modelo de gestão que leve em conta outros fatores denominados como externalidades positivas e negativas. Neste contexto, a literatura acadêmica investiga o relacionamento entre o desempenho ambiental e financeiro das empresas, isto é, a ecoeficiência organizacional, que será tratada no tópico seguinte.

\subsection{Ecoeficiência}

A ecoeficiência ganhou maior notoriedade junto ao setor empresarial a partir da publicação do livro "Mudança de Rumo", de Stephan Schmidheiny, em 1990. Fundador do Business Council for Sustainable Development - WBCSD, Schmidheiny defendia uma mudança da percepção do setor empresarial em relação às questões ambientais. Sua definição reúne elementos tidos como essenciais (progresso econômico e ambiental) para o aumento da prosperidade econômica, por meio da utilização mais eficiente dos recursos e de menos emissões nocivas para o ambiente (WBCSD, 2000a). Ao invés de se colocar exclusivamente como agente do processo de degradação, o setor empresarial pode então desempenhar um papel crucial para solucionar os desafios da sustentabilidade global. No entanto, isto só ocorreria se fosse possível fundamentar as estratégias empresariais em alternativas que conciliassem melhorias ambientais e econômicas. Por esta razão, a busca da ecoeficiência permitiria ao setor empresarial concretizar tais objetivos.

Assim, Mickwitz, Melanen, Rosenstrom e Seppala (2006) afirmam que o termo descreve a combinação entre eficiência econômica e ecológica, ou seja, reduz um impacto ecológico, enquanto adiciona valor econômico. Donaire (2009) considera que ainda prevalece a ideia que qualquer providência que venha a ser tomada em relação à variável ambiental traz consigo o aumento de despesas e o consequente acréscimo dos custos do processo produtivo.

O WBCSD (2000a) aponta que a ecoeficiência é alcançada por meio da oferta de bens e serviços a preços competitivos, que, por um lado, satisfaçam as necessidades humanas e contribuam para a qualidade de vida e, por outro, reduzam progressivamente o impacto ecológico e a intensidade de utilização de recursos ao longo do ciclo de vida, até atingirem um nível, que, pelo menos, respeite a capacidade de sustentação estimada para o planeta. VanBellen (2004) advoga que em geral, sabe-se que o sistema interage entre as diferentes dimensões, mas não se conhece especificamente o impacto dessa interação. Dessa maneira, faz-se pertinente discutir a mensuração (indicadores) e suas premissas no âmbito da ecoeficiência, itens que serão abordados na sequência deste artigo.

A ecoeficiência, tratada em âmbito organizacional, é entendida como um elemento da sustentabilidade organizacional que descreve a combinação ideal do desenvolvimento ambiental com o desenvolvimento econômico, ao passo que aborda a redução dos impactos ambientais e a promoção da utilização racional dos recursos naturais e humanos. Ainda, como visualizado na Figura 1, a ecoeficiência compõe outros objetivos que devem ser buscados para o alcance da sustentabilidade organizacional, portanto, sozinha não trará para as organizações a sustentabilidade. Portanto, se analisará, por meio deste artigo, somente a ecoeficiência e seus indicadores mais utilizados, a partir de um olhar crítico.

Revista de Gestão Social e Ambiental - RGSA, São Paulo, v. 5, n. 3, p. 183-199, set./dez. 2011. 


\subsection{Avaliação dos indicadores de ecoeficiência}

A literatura carece de definições específicas para indicadores de ecoeficiência, contudo, uma vez que é aceito um modelo de sustentabilidade, a ecoeficiência é entendida como um subsistema da sustentabilidade organizacional.

De acordo com Catarino (2003), em âmbito organizacional, indicadores consistem em informações úteis para a tomada de decisão, são ferramentas de controle e gestão. $\mathrm{O}$ autor ainda comenta que, geralmente, os indicadores são de caráter quantitativo, mas, segundo VanBellen (2006), podem também ser qualitativos, existindo autores que defendem que os mais adequados para avaliação de assuntos relacionados ao desenvolvimento sustentável deveriam ter caráter mais qualitativos, em razão das limitações que existem em relação aos indicadores quantitativos. $\mathrm{O}$ objetivo dos indicadores é agregar e quantificar informações, de modo que sua significância fique mais aparente (Vanbellen, 2006).

Para a OECD (1993), um indicador pode ser definido como um parâmetro ou um valor, que fornece informações sobre um fenômeno, tendo um significado que ultrapassa as propriedades do valor do parâmetro, e possui um significado sintético, desenvolvido para uma finalidade específica. Assim, de acordo com a OECD (1993), os indicadores têm como principais funções: a) reduzir o número de medidas e parâmetros que, normalmente, seriam necessários para uma apresentação "exata" de certa situação; e, b) simplificar o processo de comunicação pelo qual a informação dos resultados da medição é fornecida ao usuário.

Maxime, Marcotte e Arcand (2006), em seu estudo, também falando sobre organizações, mais especificamente, organizações do setor industrial de alimento e bebida, citam cinco passos para o desenvolvimento de indicadores para organizações, a saber:

- Identificação do objetivo do indicador;

- Seleção dos limites do sistema onde as entradas e saídas serão consideradas;

- Seleção do período de referência (por exemplo, anualmente);

- Identificação e quantificação de todas as entradas e saídas relevantes; e

- Cálculo, a rigor, do indicador.

Desta forma, a importância dos indicadores de sustentabilidade e, particularmente de ecoeficiência, se deve ao fato de condensarem uma enorme complexidade do sistema em questão, a uma quantidade razoável de informações significativas, e a um pequeno subconjunto de observações, informando decisões e direcionando ações. Os indicadores de sustentabilidade exprimem sua importância ao captar, de forma confiável, importantes aspectos da interação da organização com seu ambiente (Bossel, 1999).

Ainda é preciso levar em consideração, conforme explica VanBellen (2006), que as fragilidades dos indicadores de sustentabilidade se manifestam porque eles são instrumentos imperfeitos e não universalmente aplicáveis, e cada vez mais se torna necessário conhecer as particularidades dos diferentes sistemas. Embora haja grande variedade de definições, conforme aponta Siena (2008) e Moura (2002), estes autores comentam que alguns pontos são consensuais e estes são tomados por base para operacionalização do conceito de indicador de sustentabilidade: a função de medir; e de comunicar informações complexas de forma simplificada e objetiva; oferecendo contextualização em relação ao local, ao investigador e ao público a quem interessam as informações; e a visão sistêmica.

Já, as fragilidades em torno desses indicadores são descritas e resumidas por Bossel (1999) ao afirmar que atualmente nenhum indicador de sustentabilidade é adequado para fornecer informações essenciais sobre a viabilidade de um sistema e de sua taxa de mudança, e, por isso, não contribuem, como deveriam, para o desenvolvimento sustentável. Isso, provavelmente, ocorre, conforme pontuam Kranjc e Glavic (2003), porque a maior parte dos indicadores é quantitativa e quando se envolve a sustentabilidade, por sua complexidade, alguns indicadores deveriam ser qualitativos para bem expressar a variável que está em jogo.

Revista de Gestão Social e Ambiental - RGSA, São Paulo, v. 5, n. 3, p. 183-199, set./dez. 2011. 
Levando estes aspectos em consideração, para uma avaliação dos principais indicadores de ecoeficiência, conforme já mencionado na metodologia, utilizou-se os Princípios de Bellagio (apresentados na Figura 1), que, segundo Louette (2007), foram organizados em 1996 pelo International Institute for Sustainable Development - IISD em Bellagio (Itália), com o objetivo de sintetizar a percepção geral sobre os principais aspectos relacionados com a avaliação da sustentabilidade. Estes princípios abrangem todas as etapas do processo de desenvolvimento de indicadores para mensuração da sustentabilidade, que, neste artigo, são utilizados para avaliação dos indicadores de ecoeficiência.

\begin{tabular}{|c|c|}
\hline Princípio & A avaliação em direção ao desenvolvimento sustentável deve: \\
\hline $\begin{array}{l}1 \text { Guia de } \\
\text { visões e metas }\end{array}$ & $\begin{array}{l}\text { - Ser guiada por uma visão clara do desenvolvimento sustentável e metas que definem essa } \\
\text { visão. }\end{array}$ \\
\hline $\begin{array}{l}2 \text { Perspectiva } \\
\text { holística }\end{array}$ & $\begin{array}{l}\text { - Incluir visão do sistema todo e de suas partes; } \\
\text { - Considerar o bem-estar social, bem-estar ecológico e bem-estar econômico dos subsistemas; } \\
\text { seu estado atual, tendência e taxa de mudança tanto dos componentes das partes quanto da } \\
\text { interação entre as partes; } \\
\text { - Considerar as consequências positivas e negativas da atividade humana, de forma a refletir } \\
\text { os custos e benefícios para os sistemas humano e ecológico, em termos monetários e não- } \\
\text { monetários. }\end{array}$ \\
\hline $\begin{array}{l}3 \text { Elementos } \\
\text { essenciais }\end{array}$ & $\begin{array}{l}\text { - Considerar a equidade e a disparidade na população atual e entre esta e as futuras gerações, } \\
\text { lidar com a utilização de recursos, com o superconsumo e pobreza, direitos humanos e acesso } \\
\text { a serviços; } \\
\text { - Considerar as condições ecológicas das quais a vida depende; } \\
\text { - Considerar o desenvolvimento econômico e outros aspectos que não são oferecidos pelo } \\
\text { mercado e que contribuem para o bem-estar humano e social. }\end{array}$ \\
\hline $\begin{array}{l}4 \text { Escopo } \\
\text { adequado }\end{array}$ & $\begin{array}{l}\text { - Adotar um horizonte de tempo suficientemente longo para capturar as escalas de tempo } \\
\text { humano e dos ecossistemas, atendendo às necessidades das futuras gerações, bem como da } \\
\text { geração atual em termos de processo de tomada de decisão no curto prazo; } \\
\text { - Definir o espaço de estudo para abranger não apenas impactos locais, mas também o } \\
\text { impacto de longa distância sobre pessoas e ecossistemas; } \\
\text { - Construir um histórico das condições presentes e passadas para antecipar futuras condições. }\end{array}$ \\
\hline 5 Foco prático & $\begin{array}{l}\text { - Um sistema de categorias explícitas ou um sistema organizado que conecte a visão e as } \\
\text { metas com os indicadores e os critérios de avaliação; } \\
\text { - Um número limitado de questões-chave para análise; } \\
\text { - Um número de indicadores ou combinações de indicadores que sinalizem claramente o } \\
\text { progresso; } \\
\text { - Um padrão de medidas para permitir a comparação, quando possível; } \\
\text { - Comparação de valores dos indicadores com suas metas, valores de referência, limites ou } \\
\text { direção da mudança. }\end{array}$ \\
\hline $\begin{array}{l}6 \text { Abertura e } \\
\text { transparência }\end{array}$ & $\begin{array}{l}\text { - Tornar os métodos e dados usados acessíveis a todos; } \\
\text { - Deixar explícitos todos os julgamentos, suposições e incertezas de dados. }\end{array}$ \\
\hline $\begin{array}{l}7 \text { Comunicação } \\
\text { efetiva }\end{array}$ & $\begin{array}{l}\text { - Ser projetada para atender às necessidades do público e do grupo de usuários; } \\
\text { - Ser feita de forma que os indicadores e as ferramentas estimulem e engajem os tomadores de } \\
\text { decisão; } \\
\text { - Procurar a simplicidade na estrutura do sistema e utilizar linguagem clara e simples. }\end{array}$ \\
\hline $\begin{array}{l}8 \text { Ampla } \\
\text { participação }\end{array}$ & $\begin{array}{l}\text { - Obter ampla representação do público profissional, técnico e comunitário, incluindo } \\
\text { participação de jovens, mulheres e indígenas para garantir o reconhecimento dos valores, que } \\
\text { são diversos e dinâmicos; } \\
\text { - Garantir a participação dos tomadores de decisão para assegurar uma forte ligação com a } \\
\text { adoção de políticas e os resultados da ação. }\end{array}$ \\
\hline
\end{tabular}




\begin{tabular}{|c|c|}
\hline $\begin{array}{l}9 \text { Avaliação } \\
\text { constante }\end{array}$ & $\begin{array}{l}\text { - Desenvolver a capacidade de repetidas medidas para determinar tendências; } \\
\text { - Ser interativa, adaptativa e responsiva às mudanças e incertezas, porque os sistemas são } \\
\text { complexos e estão em frequente mudança; } \\
\text { - Ajustar as metas, sistemas e indicadores com as novas descobertas decorrentes do processo; } \\
\text { - Promover o desenvolvimento do aprendizado coletivo e o feedback necessário para a tomada } \\
\text { de decisão. }\end{array}$ \\
\hline $\begin{array}{l}10 \text { Capacidade } \\
\text { institucional }\end{array}$ & $\begin{array}{l}\text { - Definir clara responsabilidade e apoiar constantemente o processo de tomada de decisão; } \\
\text { - Assegurar capacidade institucional para a coleta de dados, sua manutenção e documentação; } \\
\text { - Apoiar o desenvolvimento da capacitação local de avaliação. }\end{array}$ \\
\hline
\end{tabular}

Figura 1: Princípio de Bellagio

Fonte: VanBellen (2006); Louette (2007)

Tendo em vista estes dez princípios relacionados com a avaliação da sustentabilidade, elencados em Bellagio, nota-se a complexidade de mensurar sustentabilidade e ecoeficiência. Neste contexto, Callado e Fensterseifer (2009) afirmam que os indicadores em âmbito da sustentabilidade diferem dos indicadores tradicionais de progresso econômico, social ou ambiental quando tratados de forma isolada, uma vez que estes últimos medem as mudanças de um aspecto como se fossem inteiramente independentes dos demais. Já os indicadores inerentes a sustentabilidade, assim como os indicadores de ecoeficiência, requerem uma visão integrada do mundo com indicadores multidimensionais que mostrem as inter-relações existentes entre eles, apresentando caráter sistêmico, segundo Moura (2002).

Estes indicadores, segundo VanBellen (2010), auxiliam os tomadores de decisões e o público em geral a definir os objetivos e as metas do desenvolvimento e permitem a avaliação do desenvolvimento, na medida em que alcance ou se aproxime dessas metas. Porém, para serem continuamente úteis, os indicadores precisam, conforme Callado e Fensterseifer (2009), serem periodicamente avaliados e, quando necessário, modificados e ajustados para atender às mudanças no ambiente. Além disso, os indicadores devem permitir a comparação do desempenho da empresa com o de outras empresas do setor ou empresas de outros setores.

Tendo por base os conceitos gerais em torno do desenvolvimento sustentável, da sustentabilidade organizacional, da ecoeficiência, dos indicadores e, consequentemente, da sua importância e das suas fragilidades já registradas por alguns autores, estes fornecem aporte teórico suficiente para a discussão em torno dos indicadores de ecoeficiência do WBCSD e da GRI e sua robustez.

\subsection{Apresentação dos indicadores de ecoeficiência utilizados na análise}

A ecoeficiência é elemento constituinte do desenvolvimento sustentável, logo, a utilização de instrumentos capazes de mensurá-la torna-se imprescindível no progresso rumo à sustentabilidade. Para Salgado (2007), a ecoeficiência relaciona-se com a estratégia competitiva e o desenvolvimento sustentável da empresa. O conceito de ecoeficiência em si, já prevê a definição de indicadores capazes de medir o desenvolvimento de um empreendimento. Atualmente, muitas organizações adotaram programas e projetos a fim de padronizar relatórios ambientais e medidas de ecoeficiência, alguns aplicáveis a várias empresas, outros específicos para determinados setores.

Ao realizar um levantamento, sem considerar a área de atuação das diferentes ferramentas de avaliação, VanBellen (2004) identificou dezoito metodologias ou instrumentos de avaliação da sustentabilidade mais referenciados na literatura científica. Contudo, novamente, constatou-se que há problemas conceituais e de medidas não resolvidos adequadamente pelas experiências em curso. Callado (2010), assim como Laville (2009), aponta uma extensa lista de códigos de conduta que tratam da sustentabilidade. Contudo, entre os indicadores de sustentabilidade, destaca-se mais especificamente, a fim de atender o objetivo deste trabalho, os indicadores de ecoeficiência. Salgado (2007) comenta que a necessidade de se mensurar e quantificar a ecoeficiência resultou na inserção do termo "indicadores de ecoeficiência". Assim, fixar objetivos e monitorar o desempenho com indicadores são métodos de gestão utilizados mundialmente na área de negócios, na medição

Revista de Gestão Social e Ambiental - RGSA, São Paulo, v. 5, n. 3, p. 183-199, set./dez. 2011. 
de ecoeficiência.

Neste contexto, este trabalho entende por indicadores a definição, de acordo com o escopo da OECD (1993), que diz respeito as cinco características fundamentais de Maxime et al.(2006). E o entendimento de indicadores de sustentabilidade, que podem ser aplicados a ecoeficiência, provê dos Princípios de Bellagio e da definição de Moura (2002), que possam, conforme postula Salgado (2007), permitir visualizar o desempenho ambiental e econômico das atividades e contribuir para o gerenciamento ambiental.

Portanto, foram selecionados para análise, neste trabalho, dois conjuntos de indicadores, considerados por sua relevância. O primeiro, são os indicadores criados pelo WBCSD, conselho que leva o crédito pela criação do termo ecoeficiência, sendo assim, considerado pelo seu pioneirismo em tal estudo. O segundo grupo de indicadores é aquele editado pelo GRI, que são amplamente reconhecidos a nível global e tem seus indicadores aceitos e utilizados por diversos autores, tais como Fet (2003), Schaltegger e Wagner (2005), Mickwitz et. al (2006), Maxime et al. (2006) e Piotto (2003).

O WBCSD introduziu o conceito de ecoeficiência, e publicou relatórios no ano 2000 abordando sistemas de medição e utilização de indicadores de ecoeficiência (Salgado, 2007). O WBCSD (WBCSD, 2000b) ainda lista os três objetivos centrais da ecoeficiência, para atingir mais valor, utilizando menos materiais e energia e reduzindo as emissões:

- Redução do consumo de recursos: minimização do uso de energia, materiais, água e solo, favorecendo a reciclabilidade e durabilidade do produto, fechando o ciclo dos materiais;

- Redução do impacto na natureza: minimização das emissões gasosas, descargas líquidas, eliminação de desperdícios e a dispersão de substâncias tóxicas, assim como impulsionar a utilização sustentável de recursos renováveis;

- Melhoria do valor do produto ou serviço: fornecimento de mais benefícios aos clientes, por meio da funcionalidade, flexibilidade e modularidade do produto, fornecendo serviços adicionais e concentrando-se em vender as necessidades funcionais de que, de fato, os clientes necessitam, com menos materiais e menor utilização de recursos.

A partir destes três objetivos centrais, o WBCSD (2000b) desdobra-os em sete indicadores aplicáveis para toda empresa, na melhoria da ecoeficiência, que são reforçados por Piotto (2003), e apresentados na Figura 2.

\begin{tabular}{|c|c|}
\hline \multicolumn{2}{|c|}{ INDICADORES DE ECOEFICIÊNCIA DA WBCSD } \\
\hline $\begin{array}{l}\text { - Reduzir a intensidade do consumo de } \\
\text { materiais em produtos e serviços; } \\
\text { - Reduzir a intensidade do consumo de energia } \\
\text { em produtos e serviços; } \\
\text { - Reduzir a dispersão de compostos tóxicos; }\end{array}$ & $\begin{array}{c}\text { - Promover a reciclagem; } \\
\text { - Maximizar o uso de recursos renováveis; } \\
\text { - } \quad \text { Estender a durabilidade dos produtos; e } \\
\text { Aumentar a intensidade do uso de produtos e } \\
\text { serviços. }\end{array}$ \\
\hline
\end{tabular}

Figura 2: Indicadores de ecoeficiência da WBCSD (2000b)

Fonte: WBCSD (2000b); Piotto (2003)

A segunda metodologia abordada é editada pelo GRI, que, na visão de Savitz e Weber (2007), atualmente, sem dúvida, é o principal padrão mundial para a mensuração, monitoramento e divulgação dos programas de sustentabilidade das empresas. A GRI tenta colocar os relatórios ambientais, sociais e econômicos no mesmo nível dos relatórios financeiros, em termos de rigor, clareza, exatidão, utilidade, comparabilidade e influência sobre os investidores (Savitz \& Weber, 2007).

A GRI é uma iniciativa da United Nations Enviroment Program - UNEP, datada de 1997, representando assim, o primeiro modelo para elaboração de relatórios de desempenho da

Revista de Gestão Social e Ambiental - RGSA, São Paulo, v. 5, n. 3, p. 183-199, set./dez. 2011. 
sustentabilidade nas organizações. GRI (2006) complementa que o modelo foi concebido para ser utilizado por organizações de qualquer porte, setor ou localidade, considerando as questões práticas enfrentadas por uma série de organizações, desde pequenas empresas até grupos com operações variadas e geograficamente espalhadas.

Os indicadores de desempenho de sustentabilidade da GRI estão organizados nas categorias econômica, ambiental e social, e, nestas categorias, divididos em essenciais e adicionais. Nesta pesquisa, serão considerados os indicadores das categorias econômica e ambiental, uma vez que, segundo o modelo adotado, a ecoeficiência é composta apenas destes dois pilares.

A dimensão econômica da sustentabilidade, para a GRI (2006), se refere aos impactos da organização sobre as condições econômicas de seus stakeholders e sobre os sistemas econômicos em nível local, nacional e global, como mostra a Figura 3.

\section{INDICADORES DE DESEMPENHO ECONÔMICO}

Indicador 1 - Essencial: Valor econômico direto gerado e distribuído, incluindo receitas, custos operacionais, remuneração de empregados, doações e outros investimentos na comunidade, lucros acumulados e pagamentos para provedores de capital e governos.

Indicador 2 - Essencial: Implicações financeiras e outros riscos e oportunidades para as atividades da organização, devido às mudanças climáticas.

Indicador 3 - Essencial: Cobertura das obrigações do plano de pensão de benefício definido que a organização oferece.

Indicador 4 - Essencial: Ajuda financeira significativa recebida do governo.

Indicador 5 - Adicional: Variação da proporção do salário mais baixo comparado ao salário mínimo local em unidades operacionais importantes.

Indicador 6 - Essencial: Políticas, práticas e proporção de gastos com fornecedores locais em unidades operacionais importantes.

Indicador 7 - Essencial: Procedimentos para contratação local e proporção de membros de alta gerência recrutados na comunidade local em unidades operacionais importantes.

Indicador 8 - Essencial: Desenvolvimento e impacto de investimentos em infraestrutura e serviços oferecidos, principalmente para benefício público, por meio de engajamento comercial, em espécie ou atividades pro bono.

Indicador 9 - Adicional: Identificação e descrição de impactos econômicos indiretos significativos, incluindo a extensão dos impactos.

Figura 3: Indicadores de desempenho econômico.

Fonte: GRI (2006)

A dimensão ambiental refere-se aos impactos da organização sobre sistemas naturais vivos e não-vivos, incluindo ecossistemas, terra, ar e água, de acordo com o Quadro 4.

\begin{tabular}{|l|}
\hline \multicolumn{1}{|c|}{ INDICADORES DE DESEMPENHO AMBIENTAL } \\
\hline Indicador 1 - Essencial: Materiais usados por peso ou volume. \\
\hline Indicador 2 - Essencial: Percentual dos materiais usados provenientes de reciclagem. \\
\hline Indicador 3 - Essencial: Consumo de energia direta discriminado por fonte de energia primária. \\
\hline Indicador 4 - Essencial: Consumo de energia indireta discriminado por fonte primária. \\
\hline Indicador 5 - Adicional: Energia economizada em decorrência das melhorias em conservação e eficiência. \\
\hline $\begin{array}{l}\text { Indicador } 6 \text { - Adicional: Iniciativas para fornecer produtos e serviços com baixo consumo de energia, ou que } \\
\text { usem energia gerada por recursos renováveis, e a redução na necessidade de energia resultante dessas iniciativas. }\end{array}$ \\
\hline Indicador 7 - Adicional: Iniciativas para reduzir o consumo de energia indireta e as reduções obtidas. \\
\hline Indicador 8: Total de retirada de água por fonte. \\
\hline Indicador 9 - Adicional: Fontes hídricas significativamente afetadas pela retirada de água. \\
\hline
\end{tabular}

Revista de Gestão Social e Ambiental - RGSA, São Paulo, v. 5, n. 3, p. 183-199, set./dez. 2011. 
Indicador 10 - Adicional: Percentual e volume total de água reciclada e reutilizada.

Indicador 11 - Essencial: Localização e tamanho da área possuída, arrendada ou administrada dentro de áreas protegidas, ou adjacentes a elas, e áreas de alto índice de biodiversidade fora das áreas protegidas.

Indicador 12: Descrição de impactos significativos na biodiversidade de atividades, produtos e serviços em áreas protegidas e em áreas de alto índice de biodiversidade fora das áreas protegidas.

Indicador 13 - Adicional: Habitats protegidos ou restaurados.

Indicador 14 - Adicional: Estratégias, medidas em vigor e planos futuros para a gestão de impactos na biodiversidade.

Indicador 15 - Adicional: Número de espécies na Lista Vermelha da IUCN e em listas nacionais de conservação com habitats em áreas afetadas por operações, discriminadas pelo nível de risco de extinção.

Indicador 16 - Essencial: Total de emissões, diretas e indiretas, de gases de efeito estufa, por peso.

Indicador 17 - Essencial: Outras emissões indiretas relevantes de gases de efeito estufa, por peso.

Indicador 18 - Adicional: Iniciativas para reduzir as emissões de gases causadores do efeito estufa e as reduções obtidas.

Indicador 19 - Essencial: Emissões de substâncias destruidoras da camada de ozônio, por peso.

Indicador 20 - Essencial: NOx, SOx e outras emissões atmosféricas significativas, por tipo e peso.

Indicador 21: Descarte total de água, por qualidade e destinação.

Indicador 22 - Essencial: Peso total de resíduos, por tipo e método de disposição.

Indicador 23 - Essencial: Número e volume total de derramamentos significativos.

Indicador 24 - Adicional: Peso de resíduos transportados, importados, exportados ou tratados considerados perigosos nos termos da Convenção da Basileia13 - Anexos I, II, III e VIII, e percentual de carregamentos de resíduos transportados internacionalmente.

Indicador 25 - Adicional: Identificação, tamanho, status de proteção e índice de biodiversidade de corpos d'água e habitats relacionados significativamente afetados por descartes de água e drenagem realizados pela organização relatora.

Indicador 26 - Essencial: Iniciativas para mitigar os impactos ambientais de produtos e serviços e a extensão da redução desses impactos.

Indicador 27 - Essencial: Percentual de produtos e suas embalagens recuperadas em relação ao total de produtos vendidos, por categoria de produto.

Indicador 28 - Essencial: Valor monetário de multas significativas e número total de sanções não-monetárias resultantes da não-conformidade com leis e regulamentos ambientais.

Indicador 29 - Adicional: Impactos ambientais significativos do transporte de produtos e outros bens e materiais utilizados nas operações da organização, bem como do transporte de trabalhadores.

Indicador 30 - Adicional: Total de investimentos e gastos em proteção ambiental, por tipo.

Figura 4: Indicadores de desempenho ambiental

Fonte: Gri (2006).

Embora a GRI não apresente indicadores especificamente para ecoeficiência, é muito utilizado para tal finalidade devido ao seu reconhecimento em nível global, assim, são criadas metodologias para "adaptar" estes dois conjuntos de indicadores (econômicos e ambientais) para gerarem uma medição do nível de ecoeficiência. Fet (2003), por exemplo, assim como Schaltegger e Wagner (2005), sugerem realizar uma avaliação com indicadores econômicos e ambientais de forma separada, e, posteriormente, aplicar os valores obtidos em uma equação onde se divide o resultado dos indicadores econômicos pelo resultado dos indicadores ambientais.

Tendo conhecimento dos indicadores de ecoeficiência editados pelo WBCSD e pelo GRI, estes terão sua robustez analisadas a luz dos Princípios de Bellagio. As considerações sobre indicadores tecidas pelo OCED (1993) e como estes são encarados no âmbito organizacional na visão de Catarino (2003) também serão utilizados nesta análise.

Revista de Gestão Social e Ambiental - RGSA, São Paulo, v. 5, n. 3, p. 183-199, set./dez. 2011. 


\section{METODO DE PESQUISA}

A partir do objetivo de analisar a robustez dos indicadores de ecoeficiência, assim como possíveis fragilidades existentes nestes modelos, no intuito de apontar diretrizes para a construção de novos modelos que possam aproximar-se de uma mensuração mais eficiente para as organizações, buscou-se entender o conceito de desenvolvimento sustentável, sustentabilidade e sustentabilidade organizacional, para então chegar-se a ecoeficiência. Posteriormente, fez-se necessário a compreensão do conceito de indicadores, a fim de obter suporte para a avaliação dos indicadores selecionados.

Tendo por base o trabalho de VanBellen (2006), ao analisar diferentes indicadores de sustentabilidade, optou-se utilizar os Princípios de Bellagio, que, segundo o autor, servem como orientação para avaliar e interpretar indicadores. Como suporte, utilizaram-se ainda os indicativos do OECD (1993) e de Catarino (2003), que tratam da concepção de indicadores.

Os grupos de indicadores selecionados para avaliação foram os editados pelo GRI, devido a sua ampla aceitação, e WBCSD, pelo seu pioneirismo no trato da ecoeficiência. De acordo com suas características e objetivo, a pesquisa é classificada como teórica, qualitativa e exploratória (Demo, 2000; Godoi, Silva e Bandeira-de-Mello, 2010).

\section{ANÁLISE E DISCUSSÃO DOS RESULTADOS}

Este artigo utilizou-se, dos Princípios de Bellagio (Figura 2) para realização de uma avaliação dos dois grupos de indicadores selecionados: WBCSD (Figura 3) e GRI (Figuras 4 e 5). Dando suporte aos princípios de Bellagio, buscou-se também, aporte em VanBellen (2006), OECD (1993) e Catarino (2003) para compor as discussões dos resultados.

Os indicadores do WBCSD, quando analisados a luz dos Princípios de Bellagio, não tiveram um bom desempenho, uma vez que estes indicadores não contemplam a maioria das dimensões propostas por estes princípios. Os principais motivos dessa avaliação negativa se devem ao fato de os indicadores não atenderem ao apontado pela OECD (1993) de serem concebidos como um parâmetro ou um valor, que fornece informações sobre um fenômeno. Diferentemente disto, apresentam-se como objetivos, quando o ideal seria que apresentassem possibilidades de mensuração com seus respectivos prazos. Ainda, não refletem dados como custos e benefícios, tanto monetários quanto não-monetários, e não apresentam critérios de avaliação e valores de referência, não permitindo avaliações de cunho prático. Ainda os indicadores do WBCSD não consideram os impactos das atividades no presente e no futuro (pegada ecológica) e também não consideram as dimensões temporais e as dimensões espaciais no processo de tomada de decisões.

Percebe-se que os tópicos apontados pelo WBCSD como indicadores estão distantes do próprio conceito de indicadores, de acordo com o exposto pelos autores utilizados para esta análise. Desta forma, este trabalho entende que os indicadores editados pelo WBCSD constituem um conjunto de objetivos para a ecoeficiência, uma vez que utilizam verbos, que, por sua vez, remetem a ações e ou direcionamentos, como, reduzir, promover, maximizar, estender e aumentar. Nesta análise, não é possível extrair uma informação da organização a partir do que é proposto, não fornecendo uma apresentação "exata" de certa situação, conforme aponta a OECD (1993).

Uma vez que os indicadores do WBCSD são entendidos, neste trabalho, como objetivos de ecoeficiência, não são visíveis as suposições e incertezas dos dados, bem como não fíca explícito (dando indícios da não existência) qual o método utilizado pelo WBCSD no alcance da ecoeficiência. Estes indicadores também são enfraquecidos por não trabalhar o envolvimento das partes e por não passar por avaliações constantes, uma vez que a última data de mais de dez anos. Por fim, pode-se concluir, analisando, a luz dos Princípios de Bellagio e das considerações da OECD (1993), que os indicadores do WBCSD não são robustos e talvez nem possam ser considerados indicadores, pois da forma como são apresentados são mais coerentemente compreendidos como objetivos de ecoeficiência.

Revista de Gestão Social e Ambiental - RGSA, São Paulo, v. 5, n. 3, p. 183-199, set./dez. 2011. 
Os indicadores da GRI quando analisados a luz dos Princípios de Bellagio, também não atingem a maioria das dimensões propostas pelos Princípios. Já a primeira vista, pode-se considerálos mais abrangentes, uma vez que abordam um maior número de variáveis. A divisão em indicadores essenciais e adicionais pode ser interpretada como um avanço, a fim de oferecer uma análise mais acurada para alguns tipos de organizações. Diferentemente do WBCSD, os indicadores da GRI atendem as considerações da OECD (1993) em relação ao seu conceito, pois é possível perceber que os itens apresentados são capazes de fornecer um parâmetro ou um valor. A maioria de seus indicadores fornece uma apresentação "exata" de certa situação, e tal medida pode ainda ultrapassar as propriedades diretamente associadas ao valor do parâmetro, uma vez que permitem uma avaliação qualitativa posteriormente. Somente alguns indicadores afastam-se desta exigência, uma vez que "solicitam" uma descrição de fenômenos, e, desta forma, torna-se difícil estabelecer um parâmetro, por fugir do aspecto quantitativo, não permitindo assim uma apresentação exata, conforme aponta OECD (1993). Além do mais, pode-se apontar uma comunicação efetiva e indicadores bem organizados quanto a separação de indicadores para cada dimensão (econômica, ambiental e social), utilizando uma linguagem clara e contemplando os elementos essenciais da sustentabilidade.

Entretanto, suas características negativas superam as positivas, pois, apesar de apresentar uma visão clara da ecoeficiência, não são expressas metas que orientem seu alcance. Não abordam tendências e taxas de mudança, consequências positivas e negativas, bem como monetárias e não monetárias das atividades econômicas, assim ele não proporciona uma perspectiva holística, pois mesmo que trabalhe as três dimensões da sustentabilidade, não há uma inter-relação entre elas. E, apesar de trabalhar a dimensão não apenas local, não envolve escalas temporais, o que, consequentemente, não permite prever comparações históricas.

Uma de suas principais características negativas é que por seu foco ser o de fornecer dados para elaboração de relatórios, consequentemente, os indicadores do GRI, não possuem caráter prático, metas, valores de referência e critérios de avaliação. Também, não inclui no seu instrumento de análise o interesse e as necessidades dos stakeholders. Desta forma, conclui-se que, com base nos princípios de Bellagio, os indicadores do GRI também não são robustos mediante a complexidade de medir a ecoeficiência organizacional.

Portanto, ao analisar os indicadores de ecoeficiência e ao considerar mais especificamente os indicadores do WBCSD e do Gri juntos, é importante levar em consideração que, os indicadores de ecoeficiência requerem uma visão integrada do mundo com indicadores multidimensionais que mostrem as inter-relações existentes entre as dimensões da sustentabilidade, apresentando caráter sistêmico, como afirma Moura (2002). Apesar dos indicadores do Gri mostrarem a existência de indicadores para as dimensões econômica, ambiental e social, ele não demonstra como ocorrem as inter-relações entre estes, assim como os indicadores do WBCSD não demonstram e nem ao menos fazem distinção entre as dimensões da sustentabilidade. E ainda, de acordo com Moura (2002), é necessário que todos os indicadores de ecoeficiência contemplem limites óbvios que separem o ecoeficiente do não ecoeficiente, e que tenham um valor de referência para comparação. Caso isso não aconteça, como é o caso dos indicadores em avaliação, seus usuários podem não perceber o valor da sua utilização.

Enfim, por meio deste artigo, ao analisar os indicadores visando sua possibilidade de utilização em âmbito organizacional, considera-se, conforme Catarino (2003), que quando indicadores são utilizados neste contexto devem fornecer subsídios para os processos de tomada de decisão, com base em informações importantes e resumidas em declarações concisas. Assim, observa-se que tanto os indicadores do WBCSD, quanto os indicadores do Gri não se mostram robustos neste sentido, e especula-se que isso possa ocorrer por não apresentarem um objetivo claro, e a forma como o cálculo a rigor do indicador deve ser feito, além de não possuir um período de referência e não identificar e quantificar claramente todas as entradas e saídas relevantes como orientam Maxime et al. (2006).

Revista de Gestão Social e Ambiental - RGSA, São Paulo, v. 5, n. 3, p. 183-199, set./dez. 2011. 


\section{CONSIDERAÇÕES FINAIS}

A partir do que foi considerado, observou-se que os indicadores avaliados neste artigo, mesmo sendo os mais conhecidos e utilizados, não são robustos mediante a complexidade de medir a ecoeficiência. Uma questão que se faz necessário analisar é que quando se fala em indicadores, logo se pensa em informações quantitativas, porém, no caso dos indicadores que envolvem a sustentabilidade, bem como a ecoeficiência, informações qualitativas são importantes, bem como necessárias. VanBellen (2006) afirma que existem autores que defendem os indicadores mais adequados para avaliação de experiências em assuntos relacionados ao desenvolvimento sustentável deveriam ter caráter mais qualitativo, em razão das limitações explícitas ou implícitas que existem em relação aos indicadores simplesmente numéricos.

Outra característica que os indicadores analisados não levaram em consideração na sua formulação é o peso e a necessidade de suas informações variarem de lugar para lugar e entre empresas e setores produtivos. A confirmação dessa crítica é demonstrada por VanBellen (2006) ao dizer que os indicadores de sustentabilidade são instrumentos imperfeitos e não universalmente aplicáveis, e, por isso, cada vez mais se torna necessário conhecer as particularidades dos diferentes sistemas, suas características e aplicações.

Porém, as fragilidades desses indicadores podem estar relacionadas aos poucos exemplos quantitativos de suas aplicações em estudos de casos reais, como apontam Azapagic e Perdan (2000). Além disso, estes instrumentos foram desenvolvidos com o objetivo de ser instrumentos genéricos aplicáveis a qualquer contexto e organização.

Assim, apesar das críticas apontadas, e assumindo que tais indicadores não possuem robustez suficiente para mensuração da ecoeficiência, fornecem algum subsídio para criação de indicadores adaptados ao contexto de cada organização. Desta forma, futuras pesquisas podem partir das fragilidades levantadas para trabalhar na construção de indicadores de ecoeficiência robustos, do ponto de vista do levantado na revisão bibliográfica.

\section{REFERÊNCIAS}

Azapagic, A., \& Perdan, S. (2000). Indicators of sustainable development for industry: A General Framework. Institution of Chemical Engineers, 78.

Bleischwitz, R. (2003). Cognitive and institutional perspectives of eco-efficiency. Ecological Economics, 46, 453-467.

Bossel, H. (1999). Indicators for sustainable development: theory, method, applications. Canada: International Institute for Sustainable Development.

Callado, A. L. C. (2010). Modelo de mensuração de sustentabilidade empresarial: Uma Aplicação em vinícolas localizadas na Serra Gaúcha. Tese de Doutorado, Universidade Federal do Rio Grande do Sul, Porto Alegre, RS, BR.

Callado, A. L. C., \& Fensterseifer, J. E. (2009). Indicadores de sustentabilidade. In Albuquerque, J. L. (org.). Gestão ambiental e responsabilidade social: conceitos, ferramentas e aplicações. São Paulo: Atlas.

Catarino, A. A. A. (2003). Indicadores de desempenho ambiental como instrumento de gestão e controle nos processos de licenciamento ambiental de empreendimentos de exploração $e$ produção de petróleo nas áreas offshore. Tese de Doutorado, Universidade Federal do Rio de Janeiro, Rio de Janeiro, RJ, BR.

Revista de Gestão Social e Ambiental - RGSA, São Paulo, v. 5, n. 3, p. 183-199, set./dez. 2011. 
Demo, P. (2000). Pesquisa e construção do conhecimento: metodologia científica no caminho de Habermas. Rio de Janeiro: Tempo Brasileiro.

Dyllick, T., \& Hockerts, K. (2002). Beyound the business case for corporate sustentability. Business Strategy and the environment, 11, 130-141.

Donaire, D. (2009). Gestão ambiental na empresa (2a ed.). São Paulo: Atlas.

Elkington, J. (1999). Cannibals with forks: the triple bottom line of 21 st century business. Oxford: Capstone Publishing Limited.

Fet, A. M. (2003). Eco-efficiency reporting exemplified by case studies. Clean Techn Environ Policy, 5, 232-239.

Godoi, C. K., Silva, A. B., \& Bandeira-de-Mello, R. (2010) Pesquisa qualitativa em estudos organizacionais: paradigmas, estratégias e métodos (2a ed.). São Paulo: Saraiva.

Global Reporting Initiative - Gri (2006). Diretrizes para relatórios de sustentabilidade. Boston: GRI.

Hoff, D. N. (2008) A construção do desenvolvimento sustentável através das relações entre as organizações e seus stakeholders: a proposição de uma estrutura analítica. Tese de Doutorado, Universidade Federal do Rio Grande do Sul, Porto Alegre, RS, BR.

Huppes, G., \& Ishikawa, M. (2005). A framework for quantifies eco-efficiency analysis. Journal of Industrial Ecology, 9(4), 25-41.

Kranjc, D., Glavic, P. (2003). Indicators of sustainable production. Clean Techn Environ Policy, 5.

Laville, É. (2009). A empresa verde. São Paulo: ÕTE.

Lemme, C. F. (2010). O valor gerado pela sustentabilidade corporativa. In Lins, L., \& Zylbersztajn, D. Sustentabilidade e geração de valor: a transição para o século XXI. Rio de Janeiro: Elsevier.

Louette, A. (org.). Indicadores de Nações: uma contribuição ao diálogo da sustentabilidade (1a ed.). São Paulo: WHH - Willis Harman House.

Maxime, M., Marcotte, M., \& Arcand, Y. (2006). Development of eco-efficiency indicators for the Canadian food and beverage industry. Journal of Cleaner Production, 14, 636-648.

Mickwitz, P., Melanen, M., Rosenstrom, U., \& Seppala, J. (2006) Regional eco-efficiency indicators - a participatory approach. Journal of Cleaner Production, 14, 1603-1611.

Moura, L. G. V. (2002). Indicadores para a avaliação da sustentabilidade em sistemas de produção da aGricultura familiar: o caso dos fumicultores de Agudo-RS. Tese de Doutorado, Universidade Federal do Rio Grande do Sul, Porto Alegre, RS, BR.

Munck, L., Souza, R. B. (2009a). Responsabilidade social empresarial, sustentabilidade organizacional e desenvolvimento sustentável: a proposição de uma hierarquização conceitual. Revista brasileira de estratégia - REBRAE, 2, 12-29.

. (2009b). Gestão por competências e sustentabilidade empresarial: em busca de um quadro de análise. Gestão e Sociedade, 3(6), 254-287.

Revista de Gestão Social e Ambiental - RGSA, São Paulo, v. 5, n. 3, p. 183-199, set./dez. 2011. 
. Munck, M. M. G., \& Souza, R. B. (2011, setembro). Sustentabilidade Organizacional: A Proposição de uma Framework Representativa do Agir Competente para seu Acontecimento. Anais do Encontro da Associação Nacional de Pós Graduação em Administração, Rio de Janeiro, RJ, Brasil, 35.

Organisation for Economic Co-operation and Development - OECD (1993). OECD Core set of indicators for environmental performance reviews: A synthesis report by the Group on the State of the Environment. Paris: OECD/GD.

Osorio, L. A. R., Lobato, M. O., \& Castillo, X. A. (2005). Debates on sustainable development: towards a holistic view of reality. Environment, Development and Sustainability, 7, 501-518.

Piotto, Z.C. (2003). Eco-eficiência na Indústria de Celulose e Papel: estudo de caso. Tese de Doutorado, Universidade de São Paulo, São Paulo, SP, Brasil.

Rovere, E. E., Barata, M. (2007). Prefácio. In Salgado, V. G. Indicadores de ecoeficiência e o transporte de gás natural. Rio de Janeiro: Interciência.

Salgado, V. G. (2007). Indicadores de ecoeficiência e o transporte de gás natural. Rio de Janeiro: Interciência.

Savitz, A. W., Weber, K. (2007). The triple bottom line: how today's best-run companies are achieving. San Francisco: John Wiley \& Sons Inc.

Schaltegger, T., Wagner, M. (2005). Current trends in environmental cost accounting - and its interaction with eco- efficiency performance measurement and indicators. ECO-Efficiency in Industry and Science, 18, 45-62.

Siena, O. (2008). Método para avaliar desenvolvimento sustentável: técnicas para escolha e ponderação de aspectos e dimensões. Revista Produção, 18(2).

Silva, L. S. A., Quelhas, O. L. G. (2006). Sustentabilidade empresarial e o impacto no custo de capital próprio das empresas de capital aberto. Gestão \& Produção, 13(3).

Souza, R. B. (2010). O alinhamento entre sustentabilidade e competências em contexto organizacional, Universidade Estadual de Maringá (UEM) / Universidade Estadual de Londrina (UEL), Londrina, PR, Brasil.

VanBellen, H. M. (2004). Indicadores de sustentabilidade - um levantamento dos principais sistemas de avaliação. Cadernos EBAPE.BR, 2(1).

VanBellen, H. M. (2006). Indicadores de sustentabilidade: uma análise comparativa (2a ed.). Rio Janeiro: FGV.

VanBellen, H. M. (2010). As Dimensões do Desenvolvimento: um estudo exploratório sob a perspectiva das ferramentas de avaliação. Revista de Ciências da Administração, 12(27), 153168.

Wbcsd - World Business Council for Sustainable Development. (2000a). Ecoefficiency: Creating More Value With Less impact. Geneva, Switzerland.

. (2000b). Measuring ecoefficiency: a guide to reporting company performance. Geneva, Switzerland.

Revista de Gestão Social e Ambiental - RGSA, São Paulo, v. 5, n. 3, p. 183-199, set./dez. 2011. 
World Comission On Environment And Development - Wced. (1987). Our common future: the Brundtland report. Oxford: Oxford University Press.

Data do recebimento do artigo: 15/05/2011

Data do aceite de publicação: 20/11/2011

Revista de Gestão Social e Ambiental - RGSA, São Paulo, v. 5, n. 3, p. 183-199, set./dez. 2011. 